
\title{
is Research Square \\ Controlling Nutritional Status (CONUT) score predicts worse outcomes in Chinese cardiac surgery patients
}

\section{Yan Yang}

Changzhou First People's Hospital

\section{Pei Zhang}

Changzhou First People's Hospital

\section{Hua Zhou}

Changzhou First People's Hospital

Min Yang ( $\square$ yangmin1516@czfph.com )

Department of Nephrology, The Third Affiliated Hospital of Soochow University

\section{Research}

Keywords: Malnutrition, Mortality, Cardiac surgery

Posted Date: February 10th, 2020

DOI: https://doi.org/10.21203/rs.2.22932/v1

License: (c) (i) This work is licensed under a Creative Commons Attribution 4.0 International License. Read Full License 


\section{Abstract}

Background \& Aims: Nutritional and immunological status was assessed by the Controlling Nutritional Status (CONUT) score calculated from serum albumin, total cholesterol and total peripheral lymphocyte count. The aim of this study was to determine the prognostic impact of the CONUT score on patients underwent cardiac surgery.

Methods: This is a single-center retrospective observational study in cardiac surgery patients. Baseline characteristics, comorbidities, and laboratory data were collected. The primary outcome was all-cause mortality. The secondary outcomes were postoperative complications including acute kidney injury (AKI), transient ischemic attack or stroke, reoperation for bleeding. Univariate and multivariate Cox regression were performed to investigate the association between confounding factors and outcomes. The cut-off value of CONUT score was accessed by the receiving operating characteristic curve. We used the KaplanMeier curve to compare the outcomes according to the cut-off CONUT score. The area under the curve (AUC) was used to test discriminative power of CONUT score and relevant clinical parameters.

Results: A total of 408 patients were enrolled in this study. During a median follow-up period of 72.12 months, 28 (6.9\%) patients died and 43 (10.5\%) patients occurred postoperative complications. The rate of all-cause mortality and postoperative complications was higher in the high CONUT score (>3) group than in the low CONUT score group $(p=0.002, p=0.02)$. High CONUT score (hazard ratio $[\mathrm{HR}]=2.367,95 \%$ confidence interval $[\mathrm{Cl}]=1.072$ to $5.266, \mathrm{P}=0.033$ ) was an independent predictor of all-cause mortality. The AUC of the joint probability of CONUT score, age, and hypertension in predicting all-cause mortality was 0.744 (95\% Cl: 0.687-0.775). The high CONUT score was still an independent risk factor of secondary outcomes $(\mathrm{HR}=2.316,95 \% \mathrm{Cl}=1.122$ to $4.780, \mathrm{P}=0.023)$.

Conclusion: The CONUT score was independently associated with all-cause mortality and postoperative complications in patients underwent cardiac surgery.

\section{Introduction}

Nowadays overall survival rate among patients undergoing cardiac surgery have significantly increased, reducing mortality and postoperative complications remains vital. Malnutrition is a common condition in hospitalized patients[1]. Previous studies have reported that poor nutritional status is associated with worse clinical outcomes in patients suffered from cancer, heart failure or chronic kidney diseases[2][3][4]. There are several evaluation methods of nutritional status, but some elements of malnutrition are difficult to obtain. The Controlling Nutritional Status (CONUT) score is an objective practical screening tool which is easy to perform. The CONUT score calculated from the serum albumin, peripheral lymphocyte count, and the total cholesterol. The combination of these three components can evaluate protein reserves, immune defenses, and calorie depletion[5]. Recently, the predictive value of CONUT score has been validated in operation patients suffered from peripheral arterial disease, malignant tumors and heart disease[6][7][8]. Iwakami $\mathrm{N}$ et al. investigated the association between the CONUT score and adverse 
clinical event in patients with heart failure[9]. Several studies found high CONUT score was associated with increase mortality in patients undergoing transcatheter aortic valve implantation(TAVI) or percutaneous coronary intervention[9][10]. Previous study found shrunken pore syndrome (SPS) was also associated with mortality in cardiac surgery patients [11]. However, the prognostic impact of CONUT scores and SPS has not been fully investigated in Chinese cardiac surgery patients. Therefore, this study aimed to determine the prognostic impact of malnutrition assessed by the CONUT score and SPS on patients underwent cardiac surgery.

\section{Materials And Methods}

\section{Study design and population}

We conducted a single-center retrospective observational study in cardiac surgery patients admitted to the Third Affiliated Hospital of Soochow University between February, 2011 and December, 2015. The exclusion criteria included: (1) age < 18 years old; (2) emergency operations or redo operations; (3) incidence of malignant tumor and end-stage renal disease (ESRD). This study was conducted following the principles of the Declaration of Helsinki and with the approval of the Ethics Committee at the Third Affiliated Hospital of Soochow University. Written informed consent was obtained from all patients.

\section{Data collections}

Laboratory data were obtained from the clinical chemistry department including hemoglobin, lymphocyte count, serum creatine, albumin, cystatin $\mathrm{C}$, uric acid, total cholesterol, triglyceride, high-density lipoprotein cholesterol (HDLC), low-density lipoprotein cholesterol (LDLC), C-reactive protein (CRP), $\mathrm{N}$ terminal pro B type natriuretic peptide (NT-pro BNP), cardiac troponinl (cTnl) before surgery. Perioperative data were obtained from Electronic Medical Record System. Demographics such as gender, age, and comorbidities were collected upon admission. Left ventricular ejection fraction (LVEF) was also recorded. The CONUT score was calculated based on lymphocyte count, serum albumin and total cholesterol[5] (Table 1). Estimated glomerular filtration rate (eGFR) was calculated using the Chronic Kidney Disease Epidemiology Collaboration (CKD-EPI) equation[12]. To diagnose SPS, the equation pair Caucasian Asian Pediatric Adult (CAPA)[13] and Lund Malmo Revised (LMrev)[14], based upon cystatin C and creatinine (according to www.egfr.se) was used. The tentative value of eGFR cystatin $\mathrm{C} / \mathrm{eGFR}$ creatinine ratio was 0.7 . Hypertension status was defined as blood pressure (BP) $>140 / 90 \mathrm{mmHg}$ or receiving antihypertensive drugs. Mean arterial pressure (MAP) was calculated with diastolic blood pressure plus $1 / 3$ of the pulse pressure.

\section{Clinical Outcomes and follow-up}

The primary outcome was all-cause mortality during or after cardiac surgery. The secondary outcome was postoperative complications including acute kidney injury (AKI), transient ischemic attack or stroke, reoperation for bleeding. The procedural complications were collected from the medical records. AKI was defined as increasing in $\mathrm{SCr} \geq 0.3 \mathrm{mg} / \mathrm{dl}$ within 48 hours or $\geq 50 \%$ within 7 days[15]. Clinical follow-up 
was performed via a review of medical charts and telephone interview. The occurrence of any adverse clinical events was collected.

\section{Statistical analyses}

Continuous variables with normal distribution were compared using an unpaired t-test and the results were presented as mean \pm standard deviation (SD). Non-normal distribution variables were compared by Mann-Whitney $\mathrm{U}$ test and the results presented as median (interquartile range, IQR). Categorical variables were compared by Pearson Chi-Square test and the results were presented as frequencies (percentages). Based on receiver operating characteristic (ROC) analysis results, the optimal cut-off value of CONUT score was 3. The Kaplan-Meier curves and log-rank test were conducted to estimate the survival rate of patients. To identify the independent risk factors, we performed univariate and multivariate Cox regression models. Hazard ratios (HR) and 95\% confidence intervals $(\mathrm{Cl})$ were provided. Furthermore, we also performed the area under the curve (AUC) analysis to compare models containing only CONUT score and joint models in predicting outcomes. All statistical analyses were conducted in SPSS 24.0 software. A two-sided $p$ value $<0.05$ was considered to be statistical significance.

\section{Results}

\section{Baseline characteristics}

There were 631 patients underwent cardiac surgery in our hospital. During follow-up, 178 patients withdrew. Finally, a total of 408 patients were enrolled according to the exclusion criteria. Only 12 patients (2.9\%) were diagnosed as SPS. Ninety-nine patients were classified into high CONUT score group, because their scores were more than 3 . Table 2 shows baseline clinical characteristics. The majority of patients were male, and there was significant difference in gender between two groups. Compared with low CONUT score group, patients in high CONUT score group were older. There were $196(48.0 \%)$ and 70 (17.2\%) patients having hypertension and diabetes as comorbidities respectively, the proportion was higher in high CONUT score group. Patients in high CONUT score group showed lower hemoglobin, lower serum albumin, higher serum creatinine, lower triglyceride, lower cholesterol, lower HDLC, lower LDLC, higher CRP, and lower LVEF ( $p<0.05)$. More patients met the diagnostic criteria of anemia in high CONUT score group than in low CONUT score group. There were no significant differences in MAP, current smokers, alcohol, BUN, NT-pro BNP, cTnl between two groups.

\section{Clinical outcomes}

In the present study, the median follow-up period was 72.12 months $(58.19,88.87)$, and $28(6.9 \%)$ patients achieved primary outcomes. The Kaplan-Meier total survival rate at year five was $94.9 \%$. Thirteen (13.1\%) reached the primary endpoint in high CONUT score group. As shown in Figure 1, survival rate at year five was $90.9 \%$ in the high CONUT score group compared with $96.1 \%$ in the low CONUT score group (Log rank: $p=0.002$ ). No patients diagnosed as SPS died during follow-up. Forty-three (10.5\%) patients 
occurred postoperative complications. The high CONUT group had significantly higher rate of secondary outcomes (Figure 1, Log rank: $p=0.02$ ).

\section{Related risk factors}

The correlations between baseline characteristics and all-cause mortality were analyzed by Cox regression models as well. In the univariate analysis, age $(\mathrm{HR}=1.084,95 \% \mathrm{Cl}=1.033$ to $1.138, p=0.001)$, hypertension $(\mathrm{HR}=2.975,95 \% \mathrm{Cl}=1.308$ to $6.767, p=0.009)$, high CONUT score $(\mathrm{HR}=3.179,95 \% \mathrm{Cl}=1.489$ to $6.788, p=0.003$ ) were significantly associated with an increased risk for mortality (see Table 3 ). These variables were further analyzed by a multivariate Cox regression model. High CONUT score (HR=2.367, $95 \% \mathrm{Cl}=1.072$ to $5.266, p=0.033$, Table 4$)$ and age $(\mathrm{HR}=1.062,95 \% \mathrm{Cl}=1.004$ to $1.122, p=0.034)$ remained as significant predictors of mortality. The AUC of the joint probability of CONUT score, age, and hypertension in predicting all-cause mortality was 0.744 (95\% Cl: 0.687-0.775, Figure 2).

The results of our multivariate analysis displayed that high CONUT score was still an independent risk factor of secondary outcomes ( $\mathrm{HR}=2.316,95 \% \mathrm{Cl}=1.122$ to $4.780, p=0.023$, Table 5$)$. Hypertension $(\mathrm{HR}=2.558,95 \% \mathrm{Cl}=1.101$ to $5.945, p=0.029), \mathrm{cTnl}(\mathrm{HR}=1.076,95 \% \mathrm{Cl}=1.048$ to $1.105, p<0.001), \mathrm{UA}$ $(\mathrm{HR}=1.004,95 \% \mathrm{Cl}=1.000$ to $1.007, p=0.028)$ were significantly and independently associated with secondary outcomes.

\section{Discussion}

The CONUT score is a well-known immune-nutritional index. To the best of our knowledge, the relationship between the CONUT score and mortality has not been investigated previously in Chinese patients undergoing cardiac surgery. The major finding of our study is that high CONUT scores (CONUT score $>3$ ) could be an independent predictor for all-cause mortality and postoperative complications in patients undergoing cardiac surgery.

Recently, malnutrition has been recognized as an independent predictor of mortality in patients with coronary artery disease (CAD)[16]. Ulibarri et al. reported the CONUT score to identify malnutrition in hospital population for the first time[5]. In the present study, the high CONUT score before operation was associated with higher risk of mortality and postoperative complications. Approximately $24.3 \%$ patients had high CONUT scores, the survival rate was significantly lower than the low CONUT group. In addition, the AUC of the joint probability of CONUT score, age and hypertension was the largest. According with previous study, the CONUT score was associated with increased incidence of death in asymptomatic patients with cardiac disease[17]. Poor nutritional status accessed by the CONUT score has shown to be associated with poor prognosis in patients with acute heart failure as well[9][18]. Wada $\mathrm{H}$ et al. followed 1987 patients with stable CAD undergoing percutaneous coronary intervention for 7.4 years and found that high CONUT score was related to major adverse cardiac events[10].

To date, numerous studies have evaluated the prognostic value of nutrition indicators such as in patients undergoing cardiac surgery[19]. Standard scoring systems such as the Mini-Nutritional Assessment 
(MNA), the Subjective Global Assessment (SGA), the Malnutrition Universal Screening Tool (MUST) have been regarded as significant predictors for cardiac surgical population[20][21]. However, these nutritional screening tools are based on cognitive assessments evaluated by clinicians which may cause bias. The Geriatric Nutritional Risk Index (GNRI) is calculated based on serum albumin and body weight[22]. Previous study found the CONUT score had better prognostic values than GNRI in predicting 1-year allcause mortality in patients undergoing TAVI[8]. The CONUT score contains different aspects of malnutrition via inexpensive objective markers determined from laboratory data.

Albumin is recognized as the major protein in human plasma, and it is also the most plentiful protein in the extracellular component[23]. Nutrient intake plays an important role in albumin synthesis. Therefore, malnutrition, inflammation and cachexia may lead to hypoalbuminemia[24]. Several studies detected hypoalbuminemia was associated with mortality after TAVI[25][26]. Cardiac surgery could result in an anticipated rise of inflammatory mediators[27]. Albumin, inflammatory and nutritional factors has been reported to be correlated with progression of atherosclerosis and cardiovascular disease[28][29]. Lymphocytopenia can reflect a poorly regulated immune response because of corticosteroid release[30]. Marcula $\mathrm{M}$ et al. has reported low lymphocyte counts would predict worse outcomes in patients with CAD [31]. Horwich TB et al. investigated that hypocholesterolemia was associated with marked increasing mortality in patients with heart failure[32], although it is common knowledge that hypercholesterolemia is a risk factor of CAD in the general population. Based on these reasons, the CONUT score is more appropriate to evaluate nutritional status and predict clinical outcomes in patients underwent cardiac surgery. Several studies have found that patients with chronic heart failure may be beneficial from nutritional intake[27][33]. So that we should evaluate whether nutritional interventions could improve clinical outcomes in cardiac surgery patients in follow-up studies.

A retrospective study conducted in Sweden found that SPS was associated with increasing mortality in cardiac surgery patients[11]. Nevertheless, we did not detect this association in our study. This might have been due to ethnicity differences and small sample size. SPS was originally presumed to be a risk factor for mortality in pregnant women, and similar results were obtained in men and non-pregnant women[34]. However, the incidence of SPS was low, and the cut-off value for the ratio used to diagnose SPS was uncertain. Further studies are required to provide more insight into optimal cut-off values.

However, several limitations of our study need consideration. First, it was a retrospective single-center study of Chinese population with small sample size, so that the study may suffer from a potential selection bias and a center-specific effect. Incomplete data and loss of follow-up can't be ignored. The applicability of our findings to other ethnic groups needs further validation in other cohorts. Second, we only evaluated the CONUT score before operation and did not record changes over time. Third, we could not confirm that perioperative nutritional support could improve clinical outcomes. Finally, information of cardiovascular drugs was unavailable, poor compliance might affect the clinical outcomes.

\section{Conclusion}


In summary, nutritional status evaluated by the CONUT score was independently associated with allcause mortality and postoperative complications in patients underwent cardiac surgery. Assessment of the preoperative CONUT score may provide useful prognostic value in clinical practice.

\section{Abbreviations}

CONUT Controlling Nutritional Status

AKI acute kidney injury

AUC area under the curve

HR hazard ratio

Cl confidence interval

TAVI transcatheter aortic valve implantation

SPS shrunken pore syndrome

ESRD end-stage renal disease

BUN blood urea nitrogen

UA uric acid

HDLC high-density lipoprotein cholesterol

LDLC low-density lipoprotein cholesterol

CRP C-reactive protein

NT-pro BNP N terminal pro B type natriuretic peptide

cTnl cardiac troponinl

LVEF left ventricular ejection fraction

eGFR estimated glomerular filtration rate

CKD-EPI Chronic Kidney Disease Epidemiology Collaboration

CAPA Caucasian Asian Pediatric Adult

LMrev Lund Malmo Revised

BP blood pressure 
MAP mean arterial pressure

SD standard deviation

ROC receiver operating characteristic

CAD coronary artery disease

MNA Mini-Nutritional Assessment

SGA Subjective Global Assessment

MUST Malnutrition Universal Screening Tool

GNRI Geriatric Nutritional Risk Index

\section{Declarations}

\section{Ethics approval and consent to participate}

The study was approved by the Ethics Committee of the Third Affiliated Hospital of Soochow University, China (registration number: \#24/2011), and written informed consent was obtained from all participants.

\section{Consent for publication}

Not applicable.

\section{Availability of data and materials}

The datasets used and analyzed during the current study are available from the corresponding author on reasonable request.

\section{Competing interests}

The authors declare that they have no competing interests.

\section{Funding}

This study was supported by Changzhou Sci \& Tech Program (Grant No. CJ20180027).

\section{Author contributions}

MY contributed to the conception and design of the study. YY and PZ recruited the subjects. YY and PZ analyzed the data and wrote the initial draft of the paper. $\mathrm{HZ}$ participated in its design and supervised the study. $\mathrm{HZ}$ as a professional statistician checked the statistical methods and results. All authors read and approved the final manuscript. 
Acknowledgements

Not applicable.

\section{References}

Tappenden KA, Quatrara B, Parkhurst ML, Malone AM, Fanjiang G, Ziegler TR. Critical Role of Nutrition in Improving Quality of Care: An Interdisciplinary Call to Action to Address Adult Hospital Malnutrition. Journal of the Academy of Nutrition and Dietetics, 2013, 113(9): 1219-1237.

Seo SH, Kim SE, Kang YK, Ryoo BY, Ryu MH, Jeong JH, et al. Association of nutritional status-related indices and chemotherapy-induced adverse events in gastric cancer patients. BMC Cancer, 2016, 16(1).

Honda Y, Nagai T, Iwakami N, Sugano Y, Honda S, Okada A, et al. Usefulness of Geriatric Nutritional Risk Index for Assessing Nutritional Status and Its Prognostic Impact in Patients Aged $\geq 65$ Years With Acute Heart Failure. American Journal of Cardiology, 2016, 118(4): 550-555.

Takahashi H, Ito Y, Ishii H, Aoyama T, Kamoi D, Kasuga H, et al. Geriatric nutritional risk index accurately predicts cardiovascular mortality in incident hemodialysis patients. Journal of Cardiology, 2014, 64(1): 32-36.

De Ulibarri JI, Gonzalezmadrono A, De Villar NG, Gonzalez P, Gonzalez B, Mancha A, et al. CONUT: A tool for Controlling Nutritional Status. First validation in a hospital population. Nutricion Hospitalaria, 2005, 20(1): 38-45.

Mizobuchi K, Jujo K, Minami Y, Ishida I, Nakao M, Hagiwara N. The Baseline Nutritional Status Predicts Long-Term Mortality in Patients Undergoing Endovascular Therapy. Nutrients, 2019, 11(8).

Song H, Xu B, Luo C, Zhang Z, Ma B, Jin J, et al. The prognostic value of preoperative controlling nutritional status score in non-metastatic renal cell carcinoma treated with surgery: a retrospective singleinstitution study. Cancer management and research, 2019: 7567-7575.

Okuno T, Koseki K, Nakanishi T, Sato K, Ninomiya K, Tomii D, et al. Evaluation of objective nutritional indexes as predictors of one-year outcomes after transcatheter aortic valve implantation. Journal of Cardiology, 2019, 74(1): 34-39.

Iwakami N, Nagai T, Furukawa TA, Sugano Y, Honda S, Okada A, et al. Prognostic value of malnutrition assessed by Controlling Nutritional Status score for long-term mortality in patients with acute heart failure. International Journal of Cardiology, 2017: 529-536.

Wada H, Dohi T, Miyauchi K, Doi S, Konishi H, Naito R, et al. Prognostic impact of nutritional status assessed by the Controlling Nutritional Status score in patients with stable coronary artery disease undergoing percutaneous coronary intervention. Clinical Research in Cardiology, 2017, 106(11): 875-883. 
Herou E, Dardashti A, Nozohoor S, Zindovic I, Ederoth P, Grubb A, et al. The mortality increase in cardiac surgery patients associated with shrunken pore syndrome correlates with the eGFRcystatin C/eGFRcreatinine-ratio. Scandinavian Journal of Clinical \& Laboratory Investigation, 2019, 79(3): 167173.

Stevens LA, Claybon MA, Schmid CH, Chen J, Horio M, Imai E, et al. Evaluation of the Chronic Kidney Disease Epidemiology Collaboration equation for estimating the glomerular filtration rate in multiple ethnicities. Kidney International, 2011, 79(5):555-562.

Grubb A, Horio M, Hansson LO, Björk J, Nyman U, Flodin M, et al. Generation of a new cystatin C-based estimating equation for glomerular filtration rate by use of 7 assays standardized to the international calibrator. Clin Chem. 2014;60: 974-986.

Nyman U, Grubb A, Larsson A, Hansson L, Flodin M, Nordin G, et al. The revised Lund-Malmo GFR estimating equation outperforms MDRD and CKD-EPI across GFR, age and BMI intervals in a large Swedish population. Clin Chem Lab Med. 2014;52: 815-824.

Kidney Disease. Improving Global Outcomes (KDIGO) Acute Kidney Injury Work Group: KDIGO clinical practice guideline for acute kidney injury. Kidney Int Suppl 2: 1-138, 2012.

Kunimura A, Ishii H, Uetani T, Aoki T, Harada K, Hirayama K, et al. Impact of nutritional assessment and body mass index on cardiovascular outcomes in patients with stable coronary artery disease. International Journal of Cardiology, 2017: 653-658.

Nochioka K, Sakata Y, Takahashi J, Miyata S, Miura M, Takada T, et al. Prognostic Impact of Nutritional Status in Asymptomatic Patients With Cardiac Diseases: A Report From the CHART-2 Study. Japanese Circulation Journal-english Edition, 2013, 77(9): 2318-2326.

Bermejo RA, Ferreiro RG, Roman AV, Otero IG, Kreidieh O, Sabaris PC, et al. Nutritional status is related to heart failure severity and hospital readmissions in acute heart failure. International Journal of Cardiology, 2017: 108-114.

Lopez-Delgado JC, Muñoz-Del Rio G, Flordelís-Lasierra JL, Putzu A. Nutrition in adult cardiac surgery: preoperative evaluation, management in the postoperative period and clinical implications for outcomes. Journal of Cardiothoracic and Vascular Anesthesia, 2019, 33: 3143-3162.

Lomivorotov VV, Efremov SM, Boboshko VA, Nikolaev DA, Vedernikov PE, Deryagin MN, et al. Prognostic value of nutritional screening tools for patients scheduled for cardiac surgery. Interactive Cardiovascular and Thoracic Surgery, 2013, 16(5): 612-618.

Chermesh I, Hajos J, Mashiach T,Bozhko M, Shani L, Nir RR, et al. Malnutrition in cardiac surgery: food for thought. European Journal of Preventive Cardiology, 2014, 21(4): 475-483. 
Cereda E, Pedrolli C. The geriatric nutritional risk index. Current Opinion in Clinical Nutrition and Metabolic Care, 2009, 12(1): 1-7.

Margarson M P, Soni N. Serum albumin: touchstone or totem?. Anaesthesia, 1998, 53(8): 789-803.

Von Haehling S, Doehner W, Anker SD. Nutrition, metabolism, and the complex pathophysiology of cachexia in chronic heart failure. Cardiovascular Research, 2007, 73(2): 298-309.

Yamamoto M, Shimura T, Kano S, Kagase A, Kodama A, Sago M, et al. Prognostic Value of Hypoalbuminemia After Transcatheter Aortic Valve Implantation (from the Japanese Multicenter OCEANTAVI Registry). American Journal of Cardiology, 2017, 119(5): 770-777.

Bogdan A, Barbash IM, Segev A, Fefer P, Bogdan SN, Asher E, et al. Albumin correlates with all-cause mortality in elderly patients undergoing transcatheter aortic valve implantation. Eurointervention, 2016, 12(8): e1057-e1064.

Hill A, Nesterova E, Lomivorotov V, Efremov S, Goetzenich A, Benstoem C, et al. Current Evidence about Nutrition Support in Cardiac Surgery Patients-What Do We Know?. Nutrients, 2018; doi:10.3390/nu10050597.

Ross R. Atherosclerosis - An Inflammatory Disease. The New England Journal of Medicine, 1999, 340(2): 115-126.

Turell L, Carballal S, Botti H, Radi R, Alvarez B. Oxidation of the albumin thiol to sulfenic acid and its implications in the intravascular compartment. Brazilian Journal of Medical and Biological Research, 2009, 42(4): 305-311.

Onsrud M, Thorsby E. Influence of in Vivo Hydrocortisone on Some Human Blood Lymphocyte Subpopulations. I. Effect on Natural Killer Cell Activity. Scandinavian Journal of Immunology, 1981, 13(6): 573-579.

Marcula M, Buto MF, Madaloso BA, Nunes RA, Cuoco MA, De Paula RS, et al. Lymphocyte count and prognosis in patients with heart failure. International Journal of Cardiology, 2015: 60-62.

Horwich TB, Hamilton MA, Maclellan WR, Fonarow GC. Low serum total cholesterol is associated with marked increase in mortality in advanced heart failure. Journal of Cardiac Failure, 2002, 8(4): 216-224.

Bourdelmarchasson I, Emeriau JP. Nutritional Strategy in the Management of Heart Failure in Adults. American Journal of Cardiovascular Drugs, 2001, 1(5): 363-373.

Grubb A, Lindstrom V, Jonsson M, Back S, Ahlund T, Rippe B, et al. Reduction in glomerular pore size is not restricted to pregnant women. Evidence for a new syndrome: 'Shrunken pore syndrome'. Scandinavian Journal of Clinical \& Laboratory Investigation, 2015, 75(4): 333-340. 
Tables

Table 1. Evaluation of CONUT scores

\begin{tabular}{lllll}
\hline Parameter & \multicolumn{4}{c}{ Score } \\
\hline Serum albumin, g/dl & $\geq 3.5$ & $3.0-3.4$ & $2.5-2.9$ & $<2.5$ \\
\hline Albumin score & 0 & 2 & 4 & 6 \\
Total cholesterol, mg/dl & $\geq 180$ & $140-179$ & $100-139$ & $<100$ \\
Cholesterol score & 0 & 1 & 2 & 3 \\
Total lymphocytes, count/ml & $\geq 1600$ & $1200-1599$ & $800-1199$ & $<800$ \\
\hline Lymphocytes score & 0 & 1 & 2 & 3 \\
\hline
\end{tabular}

CONUT: Controlling Nutritional Status

Table 2. Baseline clinical characteristics of 408 patients before cardiac surgery

\begin{tabular}{|c|c|c|c|c|}
\hline Clinical characteristics & Total $(\mathrm{N}=408)$ & $\begin{array}{l}\text { Low CONUT group } \\
(\mathrm{n}=309)\end{array}$ & $\begin{array}{l}\text { High CONUT group } \\
(\mathrm{n}=99)\end{array}$ & p-value \\
\hline Male (n, \%) & $246(60.3 \%)$ & $170(55.0 \%)$ & $76(76.8 \%)$ & $<0.001$ \\
\hline Age (years) & $54.9 \pm 9.2$ & $56.9 \pm 10.8$ & $61.3 \pm 11.7$ & 0.001 \\
\hline MAP (mmHg) & $97.8(96.3,102.7)$ & $96.3(91.9,101.6)$ & $97.2(89.7,101.7)$ & 0.686 \\
\hline Hypertension (n, \%) & $196(48.0 \%)$ & $138(44.7 \%)$ & $58(58.6 \%)$ & 0.016 \\
\hline Diabetes (n, \%) & $70(17.2 \%)$ & $45(14.6 \%)$ & $25(25.3 \%)$ & 0.014 \\
\hline Smoking (n, \%) & $98(24.0 \%)$ & $70(22.7 \%)$ & $28(28.3 \%)$ & 0.254 \\
\hline Alcohol (n, \%) & $24(5.9 \%)$ & $19(6.1 \%)$ & $5(5.1 \%)$ & 0.686 \\
\hline Hemoglobin $(\mathrm{g} / \mathrm{l})$ & $138.32 \pm 21.91$ & $135.16 \pm 14.85$ & $127.50 \pm 17.70$ & $<0.001$ \\
\hline Albumin (g/l) & $37.44 \pm 5.13$ & $38.52 \pm 3.76$ & $32.63 \pm 4.18$ & $<0.001$ \\
\hline $\begin{array}{l}\text { Serum creatinine } \\
(\mu \mathrm{mol} / \mathrm{l})\end{array}$ & $84.95 \pm 17.87$ & $85.76 \pm 19.70$ & $94.92 \pm 29.67$ & 0.005 \\
\hline cystatin-C (mg/l) & $0.935(0.750,1.043)$ & $0.865(0.710,0.970)$ & $1.000(0.850,1.225)$ & 0.061 \\
\hline eGFR (ml/min/1.73m2) & $83.84 \pm 15.00$ & $79.71 \pm 17.74$ & $75.53 \pm 22.02$ & 0.056 \\
\hline $\begin{array}{l}\text { Renal insufficient (n, } \\
\%)\end{array}$ & $60(14.7 \%)$ & $40(12.9 \%)$ & $20(20.2 \%)$ & 0.076 \\
\hline CAPA $(\mathrm{ml} / \mathrm{min} / 1.73 \mathrm{~m} 2)$ & $80.50(69.50,107.25)$ & $87.50(76.00,111.25)$ & $74.50(57.00,88.00)$ & 0.011 \\
\hline $\begin{array}{l}\text { LMrev } \\
(\mathrm{ml} / \mathrm{min} / 1.73 \mathrm{~m} 2)\end{array}$ & $72.71 \pm 12.07$ & $71.63 \pm 13.62$ & $65.23 \pm 16.97$ & 0.001 \\
\hline CAPA/LMrev & $1.135(0.945,1.390)$ & $1.195(1.023,1.605)$ & $1.065(0.898,1.408)$ & 0.646 \\
\hline SPS & $12(2.9 \%)$ & $10(3.2 \%)$ & $2(2.0 \%)$ & 0.533 \\
\hline Uric acid $(\mu \mathrm{mol} / \mathrm{l})$ & $384.37 \pm 104.69$ & $323.44 \pm 100.24$ & $323.14 \pm 115.45$ & 0.980 \\
\hline Triglyceride (mmol/l) & $2.01(1.50,2.71)$ & $1.82(1.36,2.59)$ & $1.39(1.19,1.91)$ & 0.001 \\
\hline Cholesterol (mmol/l) & $4.47 \pm 0.92$ & $4.40 \pm 0.99$ & $3.24 \pm 0.83$ & $<0.001$ \\
\hline CRP (mg/l) & $5.60(4.08,7.00)$ & $4.45(3.78,6.23)$ & $4.60(3.85,9.20)$ & $<0.001$ \\
\hline HDLC (mmol/l) & $1.064 \pm 0.217$ & $1.083 \pm 0.287$ & $0.900 \pm 0.243$ & $<0.001$ \\
\hline LDLC (mmol/l) & $2.416 \pm 0.587$ & $2.194 \pm 0.660$ & $1.545 \pm 0.545$ & $<0.001$ \\
\hline NT-proBNP (ng/l) & $\begin{array}{l}1155.00(405.80, \\
3490.95)\end{array}$ & $\begin{array}{l}1255.95(310.05, \\
2222.50)\end{array}$ & $\begin{array}{l}1174.05(619.15, \\
2473.88)\end{array}$ & 0.649 \\
\hline cTnI (ng/ml) & $0.0236(0.0028,2.0413)$ & $0.0185(0.0098,2.2493)$ & $0.0235(0.0100,1.6915)$ & 0.105 \\
\hline LVEF (\%) & $61(57,66)$ & $61(57,64)$ & $58(55,62)$ & 0.002 \\
\hline
\end{tabular}

CONUT: Controlling Nutritional Status; MAP: mean arterial pressure; eGFR: estimated glomerular filtration rate; CAPA: Caucasian Asian Pediatric Adult; LMrev: Lund Malmo Revised; SPS: shrunken pore syndrome; CRP: C- 
reactive protein; HDLC: high-density lipoprotein cholesterol; LDLC: low-density lipoprotein cholesterol; NTproBNP: N terminal pro B type natriuretic peptide; cTnI: cardiac troponinI; LVEF: left ventricular ejection fraction.

Table 3. Univariate Cox analysis of all-cause mortality and secondary outcomes in cardiac surgery patients.

\begin{tabular}{|c|c|c|c|c|}
\hline \multirow[t]{2}{*}{ Variables } & \multicolumn{2}{|c|}{$\begin{array}{l}\text { All-cause mortality } \\
\text { Univariate analysis }\end{array}$} & \multicolumn{2}{|c|}{$\begin{array}{c}\text { Secondary outcomes } \\
\text { Univariate analysis }\end{array}$} \\
\hline & HR $(95 \%$ CI) & $\mathrm{p}$-value & HR $(95 \%$ CI) & $\mathrm{p}$-value \\
\hline$\overline{\text { Gender (female vs male) }}$ & $0.934(0.437,1.997)$ & 0.861 & $0.963(0.522,1.777)$ & 0.905 \\
\hline Age (years) & $1.084(1.033,1.138)$ & 0.001 & $1.041(1.007,1.076)$ & 0.017 \\
\hline Hypertension & $2.975(1.308,6.767)$ & 0.009 & $2.190(1.168,4.104)$ & 0.014 \\
\hline Diabetes & $2.218(0.969,5.075)$ & 0.059 & $1.602(0.787,3.262)$ & 0.194 \\
\hline Smoking & $1.079(0.458,2.541)$ & 0.862 & $1.734(0.926,3.249)$ & 0.086 \\
\hline Alcohol & $0.608(0.082,4.474)$ & 0.625 & $1.698(0.606,4.758)$ & 0.314 \\
\hline Hemoglobin $(\mathrm{g} / \mathrm{l})$ & $0.989(0.967,1.011)$ & 0.335 & $0.995(0.977,1.014)$ & 0.627 \\
\hline Albumin $(\mathrm{g} / \mathrm{l})$ & $0.967(0.893,1.047)$ & 0.410 & $0.988(0.926,1.054)$ & 0.723 \\
\hline Serum creatinine $(\mu \mathrm{mol} / \mathrm{l})$ & $0.998(0.981,1.016)$ & 0.825 & $1.008(0.997,1.019)$ & 0.147 \\
\hline zystatin-C (mg/l) & $2.626(0.613,4.309)$ & 0.329 & $1.677(0.775,3.630)$ & 0.189 \\
\hline eGFR (ml/min/1.73m2) & $0.993(0.973,1.013)$ & 0.467 & $0.984(0.968,1.000)$ & 0.044 \\
\hline Renal insufficient & $1.943(0.825,4.574)$ & 0.129 & $2.555(1.332,4.903)$ & 0.005 \\
\hline CAPA (ml/min/1.73m2) & $0.992(0.978,1.006)$ & 0.263 & $0.993(0.981,1.004)$ & 0.204 \\
\hline LMrev $(\mathrm{ml} / \min / 1.73 \mathrm{~m} 2)$ & $0.978(0.955,1.002)$ & 0.079 & $0.977(0.958,0.996)$ & 0.019 \\
\hline ¿APA/LMrev & $0.905(0.335,2.445)$ & 0.844 & $1.090(0.504,2.361)$ & 0.826 \\
\hline टRP (mg/l) & $0.989(0.960,1.018)$ & 0.448 & $0.998(0.980,1.017)$ & 0.870 \\
\hline Uric acid $(\mu \mathrm{mol} / \mathrm{l})$ & $1.003(1.000,1.006)$ & 0.080 & $1.003(1.001,1.006)$ & 0.010 \\
\hline Triglyceride $(\mathrm{mmol} / \mathrm{l})$ & $1.097(0.864,1.392)$ & 0.448 & $1.059(0.863,1.299)$ & 0.582 \\
\hline Cholesterol (mmol/l) & $0.918(0.645,1.306)$ & 0.633 & $1.121(0.852,1.474)$ & 0.414 \\
\hline NT-proBNP (ng/l) & $1.000(1.000,1.000)$ & 0.500 & $1.000(1.000,1.000)$ & 0.007 \\
\hline$\approx \operatorname{TnI}(\mathrm{ng} / \mathrm{ml})$ & $1.005(0.935,1.081)$ & 0.886 & $1.056(1.030,1.082)$ & $<0.001$ \\
\hline LVEF (\%) & $1.011(0.945,1.081)$ & 0.754 & $1.008(0.956,1.063)$ & 0.768 \\
\hline כOUNT score $\leq 3$ & $3.179(1.489,6.788)$ & 0.003 & $2.060(1.104,3.847)$ & 0.023 \\
\hline
\end{tabular}

CONUT: Controlling Nutritional Status; eGFR: estimated glomerular filtration rate; CAPA: Caucasian Asian Pediatric Adult; LMrev: Lund Malmo Revised; CRP: C-reactive protein; NT-proBNP: N terminal pro B type natriuretic peptide; CTnI: cardiac troponinI; LVEF: left ventricular ejection fraction; HR: hazard ratios; CI: confidence intervals.

Table 4. Multivariate Cox analysis of all-cause mortality in cardiac surgery patients.

\begin{tabular}{lll}
\hline \multicolumn{1}{c}{ Variables } & \multicolumn{2}{c}{ Multivariate analysis } \\
& HR $(95 \% \mathrm{CI})$ & $\mathrm{p}$-value \\
\hline Age (year) & $1.062(1.004,1.122)$ & 0.034 \\
\hline Hypertension & $1.859(0.763,4.529)$ & 0.173 \\
\hline Diabetes & $1.246(0.519,2.987)$ & 0.623 \\
\hline COUNT score $\leq 3$ & $2.376(1.072,5.266)$ & 0.033 \\
\hline LMrev $(\mathrm{ml} / \mathrm{min} / 1.73 \mathrm{~m} 2)$ & $1.005(0.975,1.036)$ & 0.748 \\
\hline
\end{tabular}


CONUT: Controlling Nutritional Status; LMrev: Lund Malmo Revised; HR: hazard ratios; CI: confidence intervals.

Table 5. Multivariate Cox analysis of secondary outcomes in cardiac surgery patients.

\begin{tabular}{lll}
\hline \multicolumn{1}{c}{ Variables } & \multicolumn{2}{c}{ Multivariate analysis } \\
& HR $(95 \% \mathrm{CI})$ & p-value \\
\hline Age (year) & $1.019(0.974,1.066)$ & 0.417 \\
\hline Hypertension & $2.558(1.101,5.945)$ & 0.029 \\
\hline COUNT score $\leq 3$ & $2.316(1.122,4.780)$ & 0.023 \\
\hline cTnI $(\mathrm{ng} / \mathrm{ml})$ & $1.076(1.048,1.105)$ & $<0.001$ \\
\hline eGFR $(\mathrm{ml} / \mathrm{min} / 1.73 \mathrm{~m} 2)$ & $0.990(0.969,1.011)$ & 0.339 \\
\hline Uric acid $(\mu \mathrm{mol} / \mathrm{L})$ & $1.004(1.000,1.007)$ & 0.028 \\
\hline
\end{tabular}

CONUT: Controlling Nutritional Status; eGFR: estimated glomerular filtration rate; cTnI: cardiac troponinI; HR: hazard ratios; CI: confidence intervals.

\section{Figures}



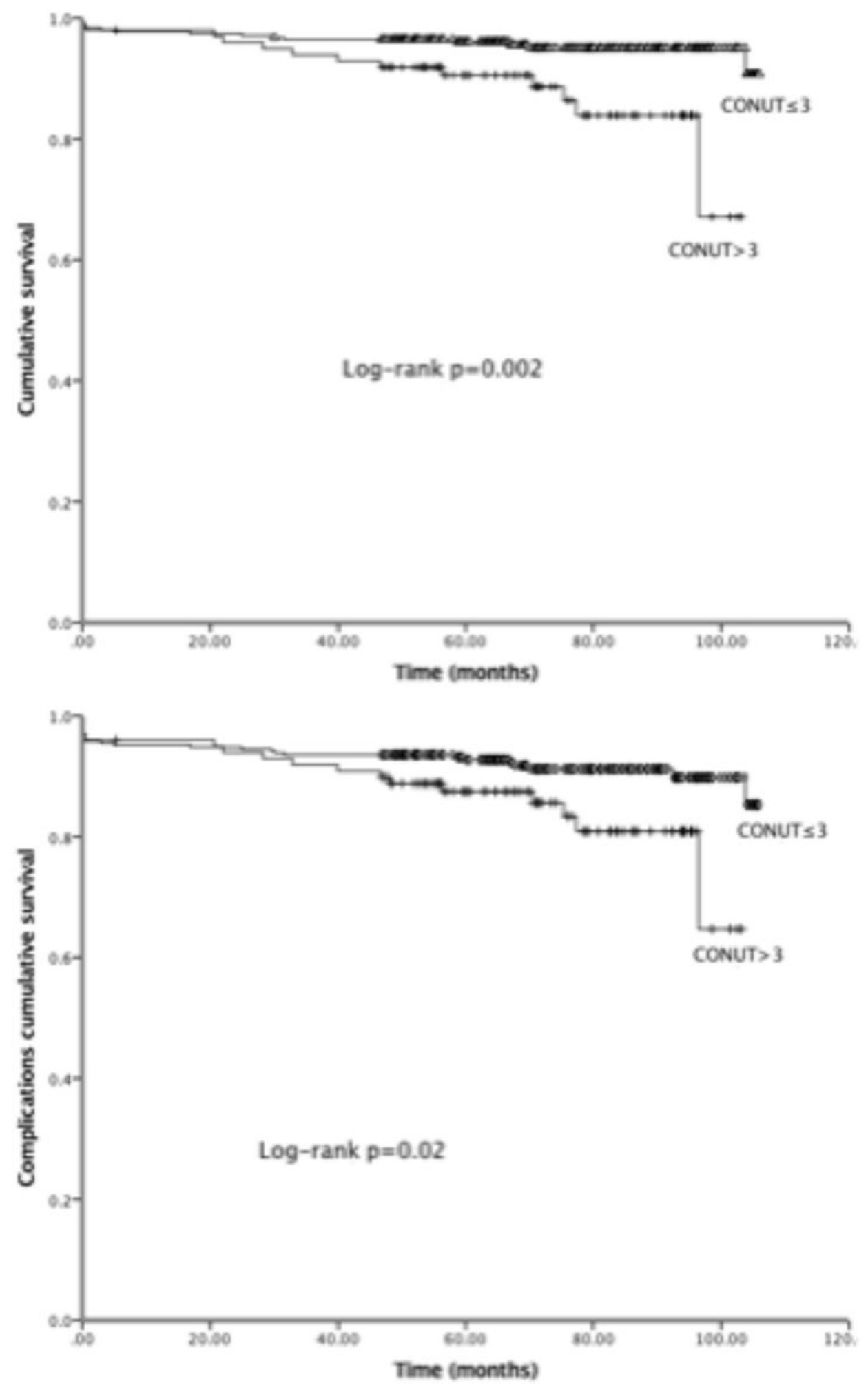

\section{Figure 2}

Kaplan-Meier curves for outcomes in patients undergoing cardiac surgery. a. all-cause mortality; b. postoperative complications. 


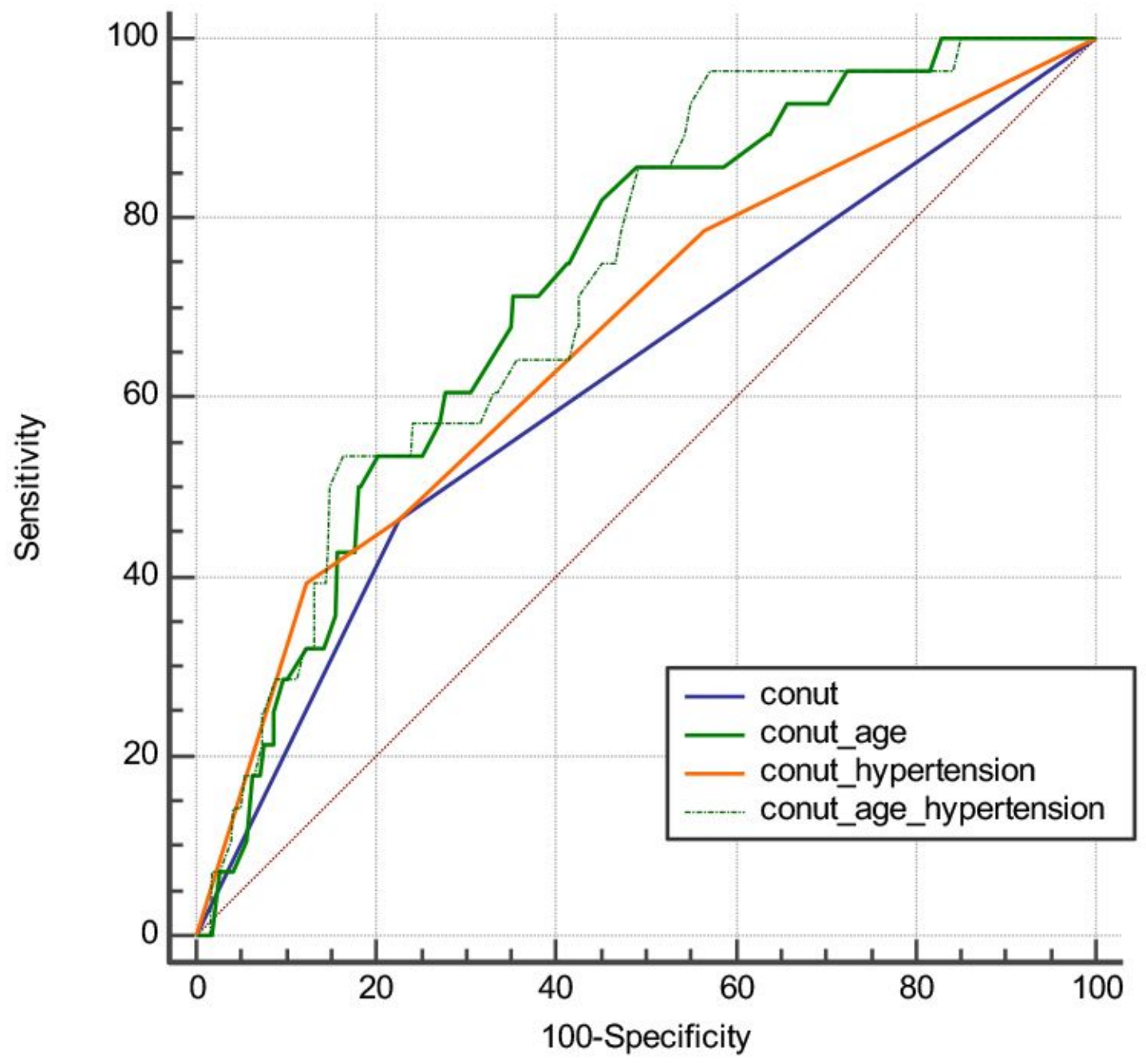

Figure 4

ROC curves of the joint probability of CONUT, age and hypertension in predicting mortality in cardiac surgery patients. 